\title{
The Role of Local Knowledge in State-and-Transition Model Development
}

\section{By Corrine Noel Knapp, Maria E. Fernandez-Gimenez, and Emily Kachergis}

$\mathrm{P}$ eople who interact with rangelands on a regular basis gain practical insights about how rangelands work by living on and working with them. This local knowledge is refined over time as individuals see the way the land responds to weather, management, and disturbances. Local knowledge (LK) is a type of knowledge "integrally linked with the lives of people, always produced in dynamic interactions among humans and between humans and nature, and constantly changing." ${ }^{1}$ LK could inform rangeland science and management to a much larger degree, but it often contributes little because the people who gain it are scattered across the landscape and there have been few attempts to systematically document and incorporate their knowledge into research or broad-scale management plans. State-and-transition models (STMs) developed for ecological sites (see Bestelmeyer et al., this issue) offer an ideal opportunity to integrate LK into durable and adaptive management tools.

STMs require information about how vegetation has changed over time in response to management and environment; however, long-term monitoring data for each ecological site are rarely available. Many recently developed STMs rely on the LK of natural resource professionals and are supported by monitoring data and research when available. ${ }^{2}$ Other long-term residents and land users often have valuable insights about rangeland dynamics and their knowledge sometimes provides the only information available. For this paper, we focus on the LK of ranchers, because they are the primary group with whom we have worked; however, we realize that other long-term residents and land users (e.g., hunters, hikers) also might hold valuable LK about rangelands.

\section{Benefits of Using LK to Develop STMs}

LK has been used in a wide range of natural resource fields from fisheries ${ }^{3}$ to nontimber forest products. ${ }^{4}$ The process of integrating LK into STMs can provide important insights about the ecosystem, engage ranchers in the process of model creation, identify critical knowledge gaps, and increase communication between ranchers, agency employees, and scientists. Despite these potential benefits, very few published studies document the incorporation of ranchers' LK into STMs.,

In comparison to ecological data collection and analysis, documenting LK can be relatively fast and provide information on a range of spatial and temporal scales. A recent STM workshop suggested that although ecological field data provide site-specific information on plant communities and the ecological attributes of existing states, LK can provide valuable information about management history and environmental context, historical or uncommon states, and vegetation dynamics at different scales. ${ }^{7}$ The types of information that LK provides would be difficult to acquire without the participation of long-term managers. For instance, it would be difficult to know the management histories of particular places, information about uncommon states and states that are no longer in existence, or how historical events influenced plant communities. Ranchers also might be able to identify practical indicators of thresholds and provide information about the interactions among ecological sites. Ecological data-driven models often rely on a process of qualitative synthesis to define states and transitions suggested by quantitative analysis of field data. LK can help to interpret field data and refine the resulting states and transitions.

The process of integrating LK into STMs helps ranchers become familiar with STMs. Participating in model development can also lead ranchers to think about landscapes in new ways (e.g., applying ecological site concepts) and incorporate these insights into their management. Ranchers often are skeptical about management practices and tools developed far from their ranch, so including LK can lend credibility and relevance to the resulting STMs. Engaging ranchers in model development allows them to participate 
actively by incorporating their knowledge into model development, instead of merely being passive recipients of information that might not reflect their experiences. Ranchers who participate in model development also can serve as liaisons to the broader ranching community, sharing information about STMs with neighbors and friends.

Including ranchers in model development can help to identify questions and concerns that local residents have about rangeland dynamics. These local management concerns may suggest research questions for universities or agencies to address. For example, a rancher involved in the development of an STM based on LK in northwest Colorado shared his hypothesis that sagebrush treatment had negative impacts on rangeland hydrology. This led to a pilot research project to determine whether the pattern he identified could be verified through systematic sampling. The process of knowledge integration serves as an opportunity for ranchers to share information and questions, and discuss unknowns with each other, agencies, and scientists.

The benefits of engaging ranchers in model creation are multiple: the models benefit from the integration of LK, the agency benefits by getting a head start on outreach and education, and ranchers benefit from exposure to a new management tool and are empowered by being included in the process.

\section{What Information Can LK Provide?}

LK has the potential to contribute several types of information to STM development, and model developers should consider the types of information that they need. Model developers can use LK to create a preliminary model to form the basis for future inventory data collection, part of the "research and reconnaissance" step of Moseley et al. (this issue). When data are available, they are often incomplete, only covering a few management/climate/soil combinations and/or states. LK could help fill in gaps by creating an inventory of historic or uncommon states as well as local disturbances and drivers. Even if a development team has access to ecological monitoring and inventory data, it could focus the integration of LK on mechanisms and timeframes for transitions or indicators of change. LK provides one way to ground-truth draft models by assessing whether they agree with local perceptions of how different soils respond to management. Finally, LK can show where there is widespread agreement on model components and where there are unknowns, questions, or contradictions that might benefit from greater attention.

\section{Options and Methods for Integrating LK}

There is a continuum of participatory processes from full participation to strategic and instrumental participation. ${ }^{8,9} \mathrm{It}$ is important that each model development team assesses its local context, including types of LK holders, resources, team skill sets, and goals before deciding how to integrate LK in model development (Table 1). The list of options we present below are meant to give model developers suggestions about how they might choose to integrate LK and are not meant as a cookbook. There is no single correct level of engagement; what works for one location and development team might not work for another. We refer to ranchers as a type of LK holder, but we recommend that model developers identify other community members with LK and consider including them in the process. In the following sections, we provide a snapshot of how LK sharing can be facilitated starting with the processes that include the most involvement from ranchers and moving towards those that include the least.

Include LK Holders on the STM Development Team Model developers can invite local ranchers to participate in the STM development team to help create an initial model based on local and professional knowledge and existing science. ${ }^{10}$ Team participants could use this draft model to identify gaps in existing knowledge and data, conflicting

\begin{tabular}{|c|c|c|c|c|c|}
\hline Method & $\begin{array}{c}\text { Number needed } \\
\text { per ecological } \\
\text { site }^{2}\end{array}$ & $\begin{array}{l}\text { Opportunity for ranchers } \\
\text { to learn about state- } \\
\text { and-transition models }\end{array}$ & $\begin{array}{l}\text { Time commitment } \\
\text { for ranchers }\end{array}$ & $\begin{array}{l}\text { Time required } \\
\text { for analysis }\end{array}$ & $\begin{array}{l}\text { Interaction among } \\
\text { participants }\end{array}$ \\
\hline Development team & $\begin{array}{l}\text { Single ongoing } \\
\text { process (4-8 } \\
\text { meetings) }\end{array}$ & High & Weeks-months & None & High \\
\hline Workshops & $1-2$ & Medium & $3-8$ hours & $4-8$ hours & Medium-High \\
\hline Interviews & $5-10$ & Medium & $1-3$ hours & $1-2$ days & None \\
\hline Focus groups & $1-2$ & Low & 2 hours & $2-6$ hours & Medium-High \\
\hline Surveys & $30-50$ & Low & 30 minutes -1 hour & $1-2$ days & None \\
\hline Feedback meeting & $1-2$ & Low & $1-2$ hours & 2-4 hours & Low-Medium \\
\hline
\end{tabular}


views about system function, and other uncertainties in the model. Based on the identified information needs, ranchers could help the team to formulate a data collection plan, participate in data analysis and interpretation, and help create a more refined model based on collected data. This level of participation requires ranchers to invest significant time in STM creation, which could mean that there are fewer participants who are willing or able to participate. Ranchers who are involved in the model development team will have a high level of involvement in all parts of the process, providing feedback on the model itself and suggesting how to make it more useful for management. The model developers will benefit from additional perspectives, insights, and information, but might spend more time organizing and facilitating the group.

\section{Hold STM Workshops}

Workshops involving multiple types of LK holders provide a focused and fast way to receive feedback on models using a variety of techniques from group discussions to hands-on modeling activities. They allow for more interaction between participants than interviews or focus groups, and diverse activities can provide more opportunities for knowledge sharing. Workshops are more successful if there is a practical and tangible goal that participants can work toward. For this method, it is a good idea to have multiple observers, including a note-taker and recorder. Team members should write down their impressions immediately after the event and compare notes later. Workshops require significant preplanning, but can be completed in four to seven hours.

\section{Individual Interviews}

One of the most effective ways to learn about LK is to interview ranchers (or other LK holders) and ask them about their understanding of and experience on the land. The number of interviews will depend on the ecological sites and their extent, the variability in production systems, and available management experience. We recommend interviewing a minimum of 5-10 knowledgeable residents to obtain information on related ecological sites. When little new information is gained from additional interviews, you have talked to enough people. Interviews should be guided by a standard list of questions to ensure consistency across all the interviews. Depending on the type of information the model development team desires, interview scripts could focus on general system dynamics or on specific questions about known states or transitions. Questions should be open-ended in order to allow participants to explain the concept of interest in more depth than a simple yes or no response.

Some ranchers might have difficulty sharing their knowledge using general or science-oriented terminology. For example, it can be challenging for them to answer abstract questions about their ecosystems such as "What are the main drivers of vegetation change in your area?" It can be very helpful to supplement sit-down interviews with conversations held out in the field, where the interview subject can refer to specific locations and tell stories about what happened there and why. We strongly recommend that interviews be audio-recorded (with the participant's permission) in addition to taking notes on paper or a laptop computer. Relying on memory alone can lead to inaccurate, poor-quality data and misrepresentation or misattribution of your informants' knowledge. The latter can undermine positive working relationships.

\section{Conduct Focus Groups}

Focus groups are small groups of 6-15 people who are invited to have an informal, semistructured conversation about a subject of interest. It is best to hold separate focus groups for different types of participants with similar backgrounds (e.g., ranchers, conservationists) so that the participants feel free to share information and opinions. Focus groups are an efficient way to gather a lot of information from a relatively large number of people in a short amount of time. Similar to an interview, the focus group discussion is guided by a list of open-ended questions. The group setting allows people to answer each question from their personal perspective, but also respond to and interact with the other participants. As in a workshop, the interactive aspect of focus groups can lead to insights that would not emerge otherwise. Focus groups could be used to develop a draft model, brainstorm an inventory of states, transitions, time scales, and indicators, or provide feedback on an existing model (Fig. 1). They are one of the most rapid of elicitation techniques, but take longer to prepare for than asking for feedback or interviewing individuals. A focus group requires a facilitator and another person to take notes. As with an interview, we strongly recommend audio-recording the focus group for accurate and complete documentation.

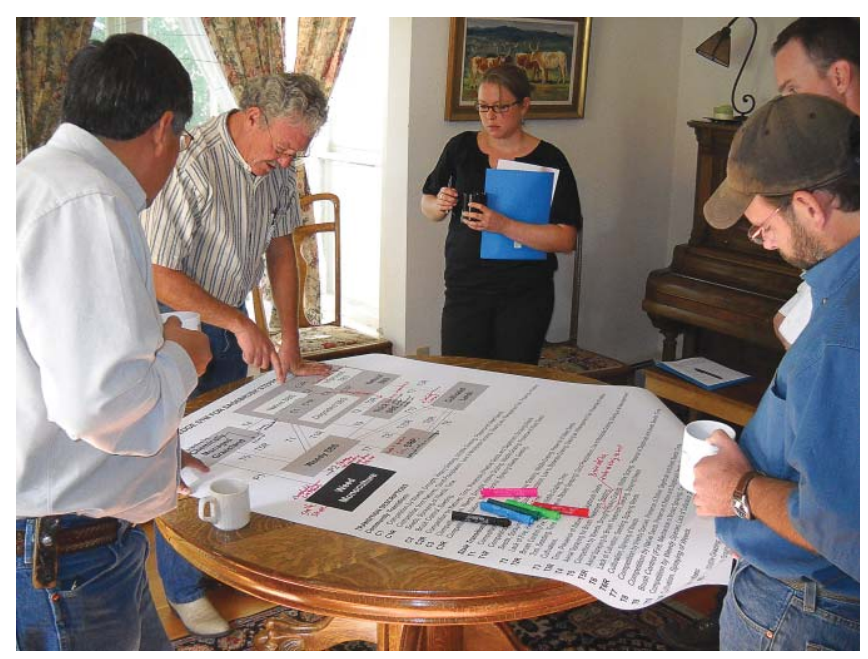

Figure 1. A group evaluating a state-and-transition model at a workshop. 


\section{Survey Questionnaires}

Survey questionnaires can be used to gather information about indicators, states, transitions, and time frames of change. The challenge with surveys is that they provide little background information or context about STMs, so respondents must have a solid understanding of STM concepts prior to filling them out. This is especially true if surveys are sent to respondents by regular mail or email with no face-to-face interaction. Few ranchers are familiar with STMs and response rates will be low if the survey material is confusing. We recommend using highly targeted surveys in combination with some kind of face-to-face participatory process. This ensures that the respondents have sufficient and similar understandings of the terminology and questions, and will generally be motivated to complete the questionnaire. The benefit of surveys used in this way is that they can provide quantitative data on LK, such as respondents' perceptions of transition probabilities and timeframes, their level of confidence in the existence of specific states, and the causes of specific transitions. Designing a valid and effective survey questionnaire takes time and expertise, but it is relatively fast to implement a survey if done in person at a workshop, focus group, or team meeting.

\section{Hold a Feedback Meeting}

Model developers can ask ranchers to provide feedback on the draft STMs. In this case, developers might host a meeting to present the draft models and solicit feedback from participants. Meetings require minimal time commitment from ranchers and allow model developers to ground-truth the models. The downside to this method is that it provides less detailed information and can be difficult or ineffective if the agency requires feedback on a large number of models in a short amount of time.

\section{Incorporating LK in STMs}

Although some methods of knowledge elicitation might directly inform model creation (meetings, development team, workshops), others can provide information that needs to be analyzed and then incorporated into models (surveys, interviews, focus groups). Local knowledge data collected using these methods is qualitative information that is rich in detail and not numerical. Analysis of qualitative data is often accomplished by summarizing notes, reflecting on key themes, and, in some situations, coding texts using qualitative data analysis software, such as NVivo. For survey data, analysis would consist of summarizing survey results using a spreadsheet or statistical package. The LK documentation and analysis techniques we have described benefit from formal training, and we recommend consulting with a trained social scientist to help design, carry out, and analyze the results of these methods. There also are several simple and accessible reference materials that would assist model developers in conducting and analyzing qualitative data (Box 1).
Box 1. Resources for documentation and analysis of local knowledge (qualitative data)

BERnARD, H. R., AND G. W. RYAN. 2009. Analyzing qualitative data: systematic approaches. London, United Kingdom: Sage Publications. $480 \mathrm{p}$.

CRESWELL, J. W. 2006. Qualitative inquiry and research design: choosing among five approaches. London, United Kingdom: Sage Publications. 416 p.

Miles, M. B., AND A. M. Huberman. 1994. An expanded sourcebook: qualitative data analysis. London, United Kingdom: Sage Publications. 352 p.

Assertions about system dynamics, whether they arise from local knowledge or field data, should be validated before integration into final models. The validation process will not resolve all questions but should identify where evidence is strong and where it is weak or contradictory. With monitoring data, replication of sample units across the landscape and repeated measurements over time are the primary means to determine which vegetation patterns and relationships apply across large areas and which might be specific to a single place. Monitoring data can be compared with LK to see if ecological sampling reflects LK, or if monitoring data is oversampling rare sites or missing rare or historical states that are no longer on the landscape. When possible, LK should be validated with field data to confirm local perceptions. Model developers can also assess the validity of LK statements by comparing individual statements with other information about the system (other LK statements, published research, monitoring data, etc.). Contradictory statements either can point to a problem with the accuracy of one of the data sources or to a need for more information. It is important that potentially conflicting information from different sources is not ignored, because it could highlight key uncertainties about the system.

Model developers should include a process to verify model components and set up ground rules for dealing with contradictory knowledge claims prior to eliciting LK. The process and ground rules could be developed either in coordination with or independent of participants. In situations where there might be skepticism about how knowledge is integrated, trust can be built by codeveloping a validation process and ground rules. If the process is completed independently of participants, it is important that it is communicated with participants so that they understand how their information/feedback will be used, how conflicting knowledge claims will be resolved or represented in the model, and who will determine which information is incorporated into any final, published STMs. These ground rules help avoid bad feelings that can occur if participants perceive that their information was not respected or valued because it does not appear in the final model. There are several processes model developers could use: 1) all types of 
knowledge could be evaluated at once and the components with the most evidence are included in the model, 2) one type of knowledge could be used to validate the other (collecting further knowledge to resolve contradictions), or 3) knowledge could be integrated collaboratively.

\section{Helpful Tips for Including LK}

Ask the Right People

A common insight from LK research is that it is important to speak with the most knowledgeable people in a community. These people can usually be located by asking other ranchers or community members to list people they respect and think are knowledgeable about the ecosystem of interest. This approach can save the development team time by narrowing down the list of potential interviewees.

\section{Be Explicit About Scale}

Ranchers manage on a landscape scale, so it is important that the development team is explicit about the scale at which the models are being developed to make sure that people share knowledge that relates to the scale of interest (landscape vs. ecological site). If developers choose to use the ecological site scale, it could be useful to have photos or ecological site maps of the area of interest, or visit specific locations on the land, so that managers can connect their knowledge to the specific ecological sites.

\section{Build a Common Vocabulary}

Long-term land managers might have different ways of talking about and understanding landscapes than range professionals. It is important to recognize these differences and not let language get in the way of sharing knowledge. Provide clear and accessible definitions of STM terms, and listen for how land managers define and understand vegetation change. Ranchers often have local names for specific plants or soils, so make sure that participants and facilitators share a common understanding about what different terms mean.

\section{Choose a Good Facilitator}

Most of the knowledge elicitation techniques that we addressed above require a facilitator. Although it might help to have an experienced facilitator, it is more important that the facilitator is a good listener and skilled at managing interactions among stakeholders. We recommend that the facilitator be someone who understands STMs, is not part of the model development team, and does not have a vested interest in the model's structure or content.

\section{Choose a Convenient Time}

Pick a time for workshops or interviews when ranchers can participate. This will vary depending on your region and the seasonality of local ranching operations. Choose a time of year and day when ranch responsibilities are low to ensure adequate participation.
Have a Plan to Deal with Conflict

Participants likely will not agree about every model component, transition driver, or timeframe. Disagreements provide valuable information about the model by highlighting areas with uncertainty and by suggesting alternative hypotheses for system behavior. As discussed previously, developers should set up ground rules and a process for model validation to guide how LK and other information sources are integrated into the final models.

\section{Respect a Different Way of Knowing}

LK resides in individual and collective memory, and ranchers' statements about vegetation change often are connected with their life or family histories and communicated through stories. Model developers must respect the lived experiences embedded in LK and recognize that many ranchers will communicate their knowledge through stories rather than providing direct answers to specific questions.

\section{Summary}

If STMs are going to be useful and credible tools for ranchers and other land users, it is important that these groups are involved in their development. By inviting stakeholders to join the STM development process, STMs benefit from additional knowledge, ranchers are given an opportunity to contribute to models and learn about them, and critical questions about the system can be highlighted. The process of knowledge integration can educate and encourage communication among ranchers, agency employees, and researchers.

\section{References}

1. Agrawal, A. 1995. Indigenous and scientific knowledge. Indigenous Knowledge and Development Monitor 3(3):3-5.

2. Suding, K. N., And R. J. Новbs. 2009. Models of ecosystem dynamics as frameworks for restoration ecology. In: R. J. Hobbs and K. N. Suding [EDs.]. New Models for Ecosystem Dynamics and Restoration. Washington, DC, USA: Island Press. p. 3-21.

3. Mackinson, S. 2001. Integrating local and scientific knowledge: an example from fisheries science. Environmental Management 27:533-545.

4. Ballard, H., and L. Huntsinger. 2006. Salal harvester local ecological knowledge, harvest practices and under-story management on the Olympic Peninsula, Washington. Human Ecology 34:529-547.

5. Bashari, H., C. Smith, and O. J. Bosch. 2008. Developing decision support tools for rangeland management by combining state and transition models and Bayesian belief networks. Agricultural Systems 99(1):23-34.

6. Knapp, C., and M. Fernandez-Gimenez. 2009. Understanding change: integrating rancher knowledge into state-andtransition models. Rangeland Ecology \& Management 62(6): 510-521.

7. Knapp, C., M. Fernandez-Gimenez, E. Kachergis, and A. Rudeen. Using participatory workshops to integrate state and transition models created with local knowledge and ecological data. Rangeland Ecology $\Xi$ Management (in press). 
8. Johnson, N., H. M. Ravnborg, O. Westermann, and K. Probst. 2001. User participation in watershed management and research. Water Policy 3:507-520.

9. Wilmsen, C., W. Elmendorf, L. Fisher, J. Ross, B. Sarathy, and G. Wells. Partnerships for empowerment: participatory research for community-based natural resource management. London, United Kingdom: Earthscan. 306 p.

10. Bestelmeyet, B. B., A. J. Tugel, G. L. Peacock, D. G. Robinett, P. L. Shaver, J. R. Brown, J. E. Herrick, H. Sanches, and K. M. Havstad. 2009. State-and-transition models for heterogeneous landscapes: a strategy for development and application. Rangeland Ecology E Management 62: $1-15$.

Authors are Research Associate, corrieknapp@yahoo.com (Knapp), Associate Professor (Fernandez-Gimenez), and Graduate Student in Ecology (Kachergis), Department of Forest, Rangeland and Watershed, Colorado State University, Fort Collins, CO 80523-1472, USA.
TRANSCENDING BORDERS

\section{LANDSCAPES and LEGENDS}

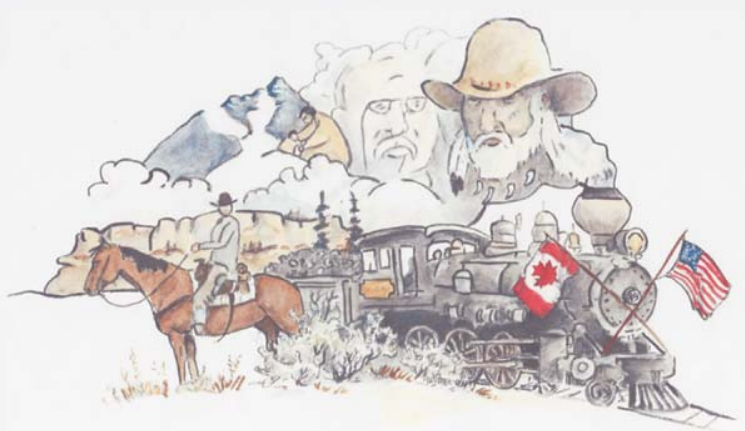

Society for Range Management Billings, Montana February 5-11 2011

http://www.rangelands.org/billings2011/ 